COMMENT OPEN

\title{
Expert consensus on the clinical application of recombinant adenovirus human $p 53$ for head and neck cancers
}

\author{
Yi Li ${ }^{1}$, Wei Guo ${ }^{2}$, Xiuqin $\mathrm{Li}^{3}$, Jianguo Zhang ${ }^{4}$, Moyi Sun ${ }^{5}$, Zhangui Tang ${ }^{6}$, Wei Ran ${ }^{7}$, Kai Yang ${ }^{8}$, Guilin Huang ${ }^{9}$ and Longjiang $\mathrm{Li}^{1 凶}$
}

The first gene therapy product, recombinant adenovirus human p53 (rAd-p53), has been approved by CFDA since 2013. During these years, most of the clinical trials and the relevant basic research were carried out by Chinese oncologists. Gendicine was proved to be a safe and promising gene therapy drug for patients who suffered from head and neck squamous cell carcinoma (HNSCC). The basic therapeutic theories of gene therapy were totally different from the traditional ones, such as surgeries or radioand chemotherapy, and the evaluation of treatment outcomes should also be changed simultaneously. However, there still existed a lot of misunderstandings about gene therapy, which resulted in improper administration, insufficient dosage calculation, and treatment cycles, and the treatment outcomes were unsatisfactory, especially for inexperienced oncologists or hospitals. Therefore, we will provide some practical guidance here on the gene therapy of rAd-p53 based on our previous research and experience, which focused on the basic theories and clinical issues, to answer the questions arising during the clinical of gene therapy and to accelerate the development of gene therapy for the benefit of patients bearing malignant tumors.

\section{INTRODUCTION}

Deoxyribonucleic acid (DNA) is a fundamental genetic substance. It is universally acknowledged that most diseases are related to genetic disorders, especially solid malignancies. If the gene transfer can be efficiently conducted to repair the disorders, it will add a new dimension to the treatment of diseases. Therefore, the gene transfer has been paid a great deal of attention. Gene therapy works by replacing defective genes with normal ones and therapeutic drugs are usually administered through viral vectors or genetically engineered microorganisms. ${ }^{1}$ Recombinant Adenovirus human p53 (rAd-p53) (Gendicine ${ }^{\mathrm{TM}}$ ) was approved by the China Food and Drug Administration (FDA) in 2003, becoming a worldwide first-line drug for the treatment of head and neck squamous cell carcinoma (HNSCC). ${ }^{2}$ However, a similar virus (Advexin, Introgen) failed to receive US FDA approval in 2008. Another clinical trial has recently been conducted using adenovirus expressing $p 53$ (Ad-p53, MultiVir) in combination with immune checkpoint inhibitors in recurrent or metastatic head and neck cancer (www.clinicaltrials.gov, NCT03544723). ${ }^{3}$ To date, a great many clinical trials have proved that it is effective enough as a wide-spectrum anticancer agent. From 2000 to 2012, most of the gene therapy-related clinical trials were performed in China, and Chinese researchers and physicians had accumulated a wealth of experience in nearly 20-year clinical practice. Ultimately, it turned out that gene therapy is a safe and promising approach for HNSCC depending on optimal treatment design and drug administration. ${ }^{4}$
Therefore, we will provide some practical guidance on the clinical application of gene therapy for head and neck cancers.

\section{THE BASIC FUNCTION OF P53 GENE}

$P 53$ is closely associated with multifaceted biological functions, including but not limited to DNA replication, modification and repair, cell cycle regulation, cell-fate determination, angiogenesis, anti-infection and immunity. ${ }^{5,6}$ P53 plays an important part in cell cycle by regulating cell cycle checkpoints of $G 1 / S, S$, and $G 2 / M$ phases to bring a halt to cell cycle progression and DNA replication, while allowing DNA repair. Both cell cycle arrest and cell apoptosis are p53-mediated canonical tumor-suppressive effects.,8 P53mediated apoptosis is realized by activating the caspase cascades and shifting the balance of the $\mathrm{Bcl}-2$ family through the mitochondria intrinsic pathway. ${ }^{9}$ Besides apoptosis, recent studies discovered p53 suppresses cystine uptake and sensitizes cells to ferroptosis via inhibiting the expression of a key component of the cystine/glutamate antiporter, SLC7A $11 .{ }^{10}$ It was also found that p53 inhibited cancer metastasis by transactivating miR-200c and miR-34a to interfere the key epithelial-mesenchymal transition drivers to abrogate this process. ${ }^{11,12}$ P53 also restrains c-Myc signaling and Yap-Hippo pathway via transactivating miR-145 and Ptpn14, respectively, to repress cancer progression. ${ }^{13,14}$ Moreover, p53 is found to regulate autophagy by activating autophagy-related genes (such as AMPK, DRAM, SESN1, and SESN2), thus inhibiting tumor

\footnotetext{
${ }^{1}$ State Key Laboratory of Oral Diseases \& National Clinical Research Center for Oral Diseases \& Department of Head and Neck Oncology, West China Hospital of Stomatology, Sichuan University, Chengdu, China; ${ }^{2}$ Department of Oromaxillofacial Head and Neck Oncology, Shanghai Ninth People's Hospital, College of Stomatology, Shanghai Jiao Tong University School of Medicine, Shanghai, China; ${ }^{3}$ Department of Obstetrics and Gynecology, Shengjing Hospital China Medical University, Shenyang, China; ${ }^{4}$ Department of Oral and Maxillofacial Surgery, School and Hospital of Stomatology, Peking University, Beijing, China; ${ }^{5}$ Department of Oral and Maxillofacial Surgery, The Third Affiliated Hospital, Air

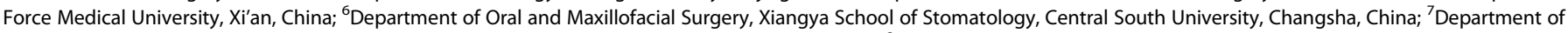
Oral and Maxillofacial Surgery, First Affiliated Hospital of Sun Yat-sen University, Guangzhou, China; ${ }^{8}$ Department of Oral and Maxillofacial Surgery, The First Affiliated Hospital of Chongqing Medical University, Chongqing, China and ${ }^{9}$ Department of Oral and Maxillofacial Surgery, Stomatological Hospital, Zunyi Medical University, Zunyi, China Correspondence: Longjiang Li (muzili63@163.com)
}

Received: 23 June 2021 Revised: 5 October 2021 Accepted: 26 October 2021

Published online: 16 November 2021 


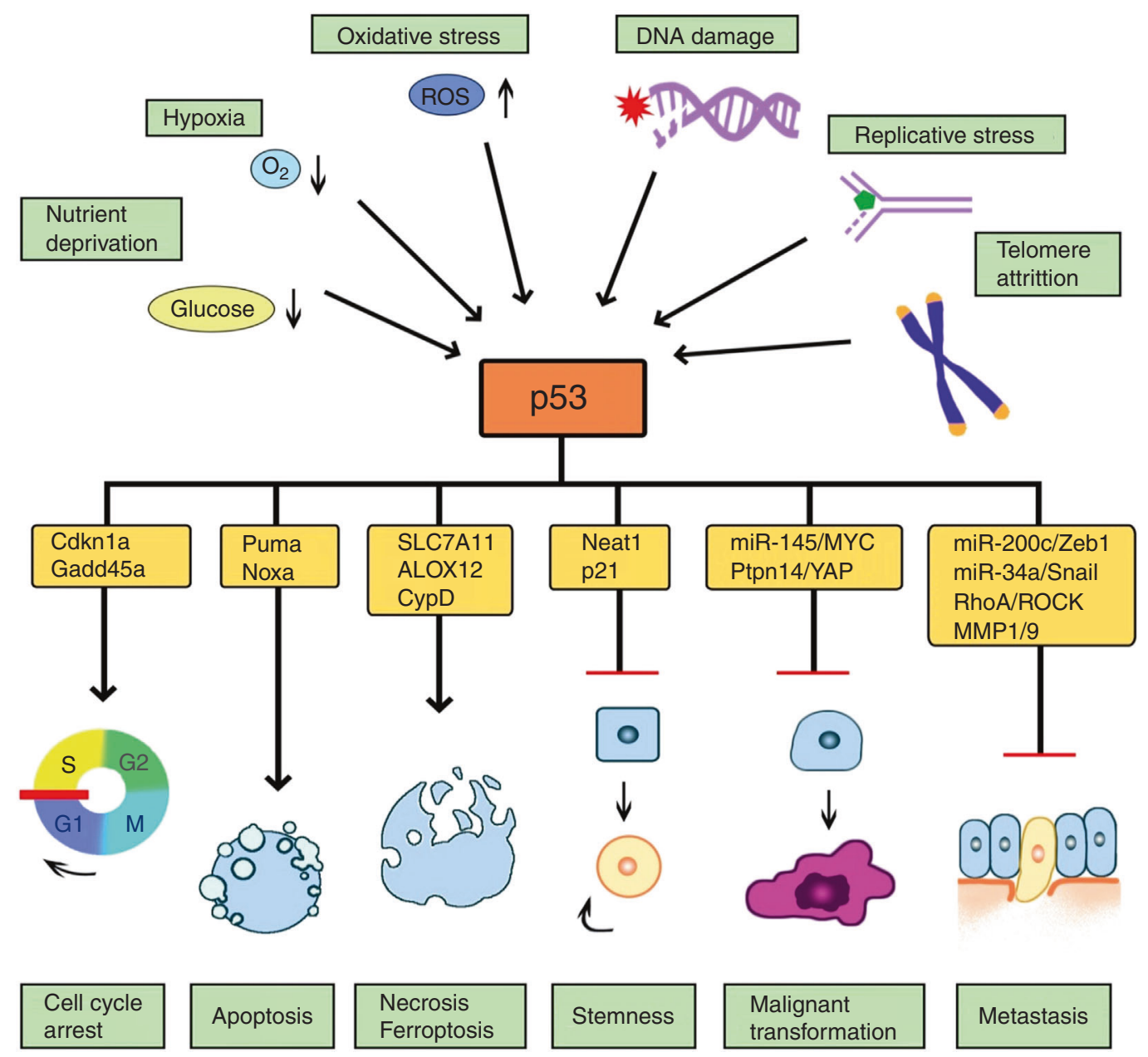

Fig. 1 Biological functions of $\mathrm{p} 53$

growth and proliferation. ${ }^{15}$ In addition, p53 may play indirect roles in regulating the downstream functional proteins as a tumor suppressor by restraining stemness via miR-34a and Neat1, ${ }^{16}$ inhibiting angiogenesis, ${ }^{17}$ mediating cellular senescence, ${ }^{18}$ generating a bystander effect, ${ }^{19}$ modulating tumor microenvironment, ${ }^{20}$ inducing immune responses ${ }^{21}$, and so on. The basic mechanisms underlying these canonical and non-canonical functions of p53 are briefly summarized below (Fig. 1).

\section{EXPERT GROUP}

The famous head and neck oncologists from the committee of integrative medicine of oral and maxillofacial oncology of the Chinese Anti-Cancer Association were invited to discuss and conduct this expert consensus. The ten participants were from nine different institutions all over China and all of them worked clinically. The participants' backgrounds included head and neck surgery and medical oncology. The participants had an average 23.9 years of research experience in the field of clinical studies of head and neck oncology or related areas since their doctoral degree. A workgroup of two members (L.J.L. and Y.L.) prepared the draft and an online meeting was convoked to invite all the attendees to discuss the contents and form the final version of the present expert consensus.

\section{GENE TRANSFER}

Gene transfer refers to a biomedical technology through which normal and therapeutic genes can be transferred into target cells in order to correct genetic disorders and consequently achieve

therapeutic purposes. ${ }^{22}$ One of the correcting methods is the in situ reparation of genetic defects, another is the replacement of tumor suppressor genes with normal and functional genes from the patient's genome. Different from traditional therapeutic methods, gene therapy is targeted at abnormal genes. It is verified that several approaches of gene therapy have been successfully performed to transfer normal genes into target cells, including biological, physical and chemical transfer. ${ }^{23}$ Further adenoviruses are shown to be the most common vectors of gene transfer. $^{24}$ With the development of biotechnologies and a growing understanding of genetic diseases, a growing number of types of gene therapy have flourished, including gene correction, gene replacement, gene augmentation, gene deactivation, suicide gene, immunotherapy, and drug resistance. ${ }^{25}$

\section{THE SELECTION OF TARGET GENES AND VECTOR CONSTRUCTION}

P53 may be an ideal target of gene transfer, as the $p 53$ gene plays a vital part in anti-tumor responses in normal cells and its mutations are frequently observed in solid malignancies. It is the momentous role of $p 53$ in the occurrence and development of malignancies that makes it a key gene of anti-tumor gene therapy. Exactly taking advantage of the core role of the $p 53$ gene, Chinese researchers produced $r A d-p 53$, which transferred a single gene into tumor cells and then gave rise to a series of anti-tumor biological capabilities so as to achieve the treatment of malignant tumors. rAd-p53 restricts its viral infectivity to a single cell cycle and the adenoviral genome does not fuse with host genomic DNA nor does it impair normal cell. ${ }^{26}$ 
The $\mathrm{rAd}-p 53$ is a recombinant human serotype 5 adenovirus where a human wild-type $p 53$ expression cassette takes the place of $E 1$ region. $r A d-p 53$ is generated by proprietary production cell lines grown in bioreactors. rAd-p53 generated by the bioreactor is further treated and purified by chromatography to obtain the injection reagent. ${ }^{27}$ Recombinant adenovirus is the most widely used viral vector at present, by virtue of its favorable characteristics for gene therapy, for instance, high gene transfer efficiency, large gene carrying capacity, and mild cytotoxicity. ${ }^{28}$

\section{INDICATIONS AND CONTRAINDICATIONS OF RAD-P53 IN THE TREATMENT OF HNSCC}

Indications

Numerous studies have confirmed that $p 53$ mutations occurred in more than $70 \%$ of HNSCC and in more than $50 \%$ of other malignancies throughout the body. Therefore, $p 53$ gene mutation is viewed as a common phenomenon in human solid malignancies. All cancer patients with p53 gene mutations detected before treatment (concurrent with the biopsy diagnosis) are in the indication group of rAd-p53 treatment. It is commonly used in patients with locally advanced stage, metastasis, or/and recurrence, in combination with radiotherapy, thermotherapy, chemotherapy, biological therapy, and other types of therapies.

\section{Contraindications}

Significant cardiac, hepatic, renal, pulmonary, or other major organ failures; severe myelosuppression and severe coagulation dysfunction that cannot be corrected; uncontrolled hypertension and diabetes mellitus; neuropsychiatric or immune system disorders; pregnant or lactating women; Eastern Cooperative Oncology Group (ECOG) physical status score $\geq 3$ or Karnofsky behavioral status score $<70$; expected survival time $<3$ months; other patients who are not suitable for rAd-p53 therapy.

\section{ADMINISTRATION METHODS AND THERAPEUTIC SCHEMES}

Dosage calculation

The efficient transduction of gene therapy reagents relies on a high multiplicity of infection (MOI), which is defined as the proportion of agents to the target cells. In other words, the virus particles are supposed to be adequate enough to transfer into target cells. However, it is of vital significance to obtain an optimal $\mathrm{MOI}$ in the administration of gene therapeutic drugs, for an excess of virus particles can result in severe hepatic damage and even individual death. ${ }^{29}$ Conventional dose determination methods utilized in chemotherapy, which depend on the body surface area or body weight, may be inappropriate for gene therapy. The basic elements of dosage calculation of gene therapy reagents involve malignant cell quantity calculated from the tumor volume and the mean cell density in the nidus, as well as the routes of administration. The tumor volume was measured by computed tomography $(\mathrm{CT})$ or magnetic resonance imaging (MRI) before therapy, the total number of cells per cubic centimeter of a solid malignant tumor is $1 \times 10^{9}$, the MOI for the one-time successful transduction of $80 \%$ of tumor cells is 100 , and the dosage calculation formula of local administration is as follows. ${ }^{30}$

$$
\begin{array}{r}
\text { Dosage per administration }(\mathrm{vp})=\text { tumor volume }\left(\mathrm{cm}^{3}\right) \times \\
\text { mean cell density }\left(10^{9}\right) \times \mathrm{MOI}(100)
\end{array}
$$

Routes of administration

To ensure the dose concentration in the tumor area, it is ideal to increase the therapeutic dose of gene therapy through local administration. Human rAd-p53 is usually administered locally, intra-tumoral injection, intra-arterial infusion, and intracavitary perfusion included. ${ }^{31}$
Intra-tumoral injection. The most important point of intratumoral injection lies in the three dimensions of tumors, which means that we should not only divide a tumor into nine gridded surfaces but also conduct a zoning design considering the depth of invasion. Therefore, CT or MRI images should be carefully read and fully comprehended before injection. Three-dimensional reconstruction is of great help to formulate treatment plans. Superficial tumors can be injected directly, while ultrasoundguided injection can be utilized for deep tumors. According to the size of tumors, the preparation should be diluted with an appropriate amount of $0.9 \%$ saline before injection to ensure that the amount of virus particles per milliliter is not $<1 \times 10^{11} \mathrm{vp}$.

Prior to multipoint intra-tumoral injections, local block anesthesia is advised for eliminating pain in the local area. According to the optimal infection titer of rAd-p53 $(\mathrm{MOI}=100)$ and the single maximal toleration dosage, the injection specification is determined as $1 \times 10^{11} \mathrm{vp} / \mathrm{cm}^{3} / \mathrm{ml}$, which means one injection site for $1 \mathrm{~cm}^{3}$ and $1 \mathrm{ml} \mathrm{rAd}-p 53$ solution for one site. ${ }^{32}$ The injection needle should be inserted vertically into the tumor nidus. The injection should cover the entire tumor and the para-cancer tissues within about $0.5 \mathrm{~cm}$.

Arterial infusion

Each anatomical area of the head and neck is supplied by a welldefined branch system of the external carotid artery, which is quite conducive to the utilization of arterial drug delivery. Prior to administration, a retrograde arterial cannula of the superficial temporal artery is performed under local anesthesia. The catheter should be put into the artery supplying the region to be treated and the drug delivery equipment should be placed subcutaneously in the temporal area. ${ }^{33}$ The dosage is estimated depending on the tumor volume and the preparation was diluted to $30 \mathrm{ml}$ with $0.9 \%$ saline, and the infusion was controlled by a micropump within $30 \mathrm{~min}$ to ensure the duration of transduction.

Other routes of administration. Human rAd-p53 is mainly used for the treatment of head and neck malignancies, through the abovementioned intra-tumoral injection and arterial infusion. As for the treatment of malignancies in other parts, there is an expanded indication. Clinical experience showed that interventional administration combined with hepatic artery embolization was suitable for the treatment of hepatocellular carcinoma, and intraperitoneal or thoracic perfusion is suitable for the treatment of metastatic carcinoma in the abdominal or thoracic cavity, malignant pleural effusion, or ascites. Notably, intravenous administration should not be adopted, as the dosage has to be increased (the maximum single dosage of Gendicine should not exceed $6 \times 10^{12} \mathrm{vp}$ ) to achieve the therapeutic outcomes on account of the broad distribution of products. rAd-p53 lacks the targeting ability to the tumor cells and the higher dosage will not increase the concentration significantly in the tumor focuses, but may lead to serious complications, such as liver failure or damage of pulmonary function, which might be caused by the aggregation of rAd-p53 in the lung and liver during intravenous administration.

\section{Administration cycles}

Studies have demonstrated that WT-p53 and its downstream factors peak at $72 \mathrm{~h}$ after successful transduction and then decline. $^{26,30}$ Due to the rapid depletion of exogenous genes, the effects of gene therapy do not last long enough to maintain high levels of functional proteins. Therefore, transduction should be repeated every $72 \mathrm{~h}$ after the first administration, to keep the continuous expression of $p 53$ to guarantee practical self-repair of tumor cells. Frequent administration is indispensable in gene therapy, as it is difficult to effectively and completely modify all malignant cells just in one transduction, and thus a series of cycles are necessary for accomplishing the aim. Nevertheless, the association between the expression and function of target genes 


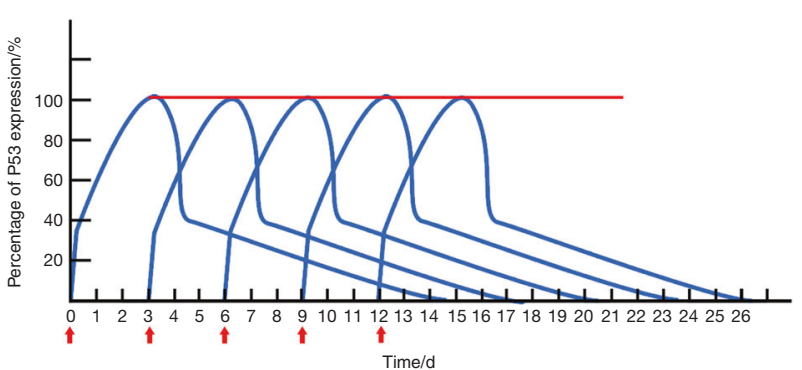

Fig. 2 Diagram of $p 53$ expression in the cancer cells during the periodical transferring of exogenous wt-p53 gene. Note: "the red line" indicating the continuous high expression of $p 53$ proteins; "the red arrows" indicating the time points for the administrations of rAd- $p 53$, the treatment cycles are 3 days between each administration point

remains unclear. Theoretically, it should be ensured that target genes are sustainedly and highly expressed in cancer cells to impose an influence on the cytogenetic material. As a consequence, the treatment of human rAd-p53 for HNSCC should include a regimen of at least five cycles of transduction to guarantee effective outcomes (Fig. 2).

\section{Therapeutic schemes}

In combination with radiotherapy and chemotherapy. Gene therapy can play a vital role in combination therapies. ${ }^{34}$ Cells have a precise regulatory system in response to radiation-induced or chemical-induced DNA damage, and the $p 53$ gene plays a crucial part in the DNA repair system. ${ }^{3,36}$ The p53-dependent gene activation profile can drive tumor cells towards apoptosis or cell cycle arrest, and this pattern relies on the posttranslational modification of $p 53 .{ }^{37,38}$

Based on these mechanisms, it is possible to kill tumor cells to the maximum extent and at the same time protecting normal ones. Hence, the $p 53$ gene can be regarded as a potential therapeutic target to reduce adverse reactions. ${ }^{39,40}$ Besides, p53 influences the chemosensitivity of tumor cells through senescence and bystander effects, which imply that some tumor cells not directly treated with chemotherapy or radiotherapy can be killed or damaged through the diffusion of soluble pro-death factors in the target cells. Consistent with radiotherapy, chemotherapy drugs are primarily targeted at damaging DNA. ${ }^{41,42}$ In both chemotherapy and radiotherapy, the p53-dependent signaling pathway activated by DNA damage exhibits similarities and the $W T-p 53$ gene plays a crucial part in the induction of apoptosis. Therefore, it is of great importance to combine chemoradiotherapy with rAd-p53 gene therapy to repair damaged DNA and lessen the therapeutic resistance. In addition, WT-p53 protein is able to attenuate the poisonousness of chemotherapy agents through some ways: (1) the interaction between p53 protein and DNA helicase; (2) p53 increases ribonuclease reductase; and/or (3) $3^{\prime} \rightarrow 5^{\prime}$ exonuclease activity of p53 protein. ${ }^{43,44}$ It is indicated that the combination application of $\mathrm{rAd}-\mathrm{p} 53$ significantly facilitates the inhibition of chemotherapy agents on the proliferation and apoptosis of malignant cells. ${ }^{21,45}$

The mechanisms of chemoradiotherapy and gene therapy are fairly distinct and these therapeutic strategies can complement each other. In clinical practice, the first step is the administration of gene therapy to reconstruct the p53 pathway and to concentrate cancer cells in the G1 phase, which constitutes the so-called "cell preparation," then followed by chemotherapy and/ or radiotherapy starting on day 3 after the first administration of human rAd-p53. With cisplatin and 5-Fu or docetaxel, and cisplatin and 5-Fu as chemotherapy regimens and external irradiation for radiotherapy, gene therapy drugs still keep administered in cycles during chemotherapy and/or radiotherapy. Such a procedural therapeutic scheme allows achieving better results, while minimizing treatment resistance. ${ }^{46,47}$

In combination with thermotherapy. As thermotherapy has become an adjuvant treatment for cancers, the combination of gene therapy with thermotherapy constitutes an effective therapeutic strategy. The transduction of the normal p53 gene using a viral vector can inhibit and reverse the malignant phenotype of tumors and induce thermal sensitization or radiosensitization, which is a novel strategy for converting a heatresistant or radiation-resistant phenotype into a thermosensitive or radiosensitive phenotype. ${ }^{48}$ In clinical practice, after two cycles of the intra-tumoral injection of $\mathrm{rAd}-p 53$, superficial tumors were suggested to be treated with a $915 \mathrm{MHz}$ microwave device at $43^{\circ} \mathrm{C}-44^{\circ} \mathrm{C}$ for $1 \mathrm{~h}$ of thermotherapy 2 days a week, and a $41 \mathrm{MHz}$ radiofrequency machine at $42^{\circ} \mathrm{C}-43^{\circ} \mathrm{C}$ for $1 \mathrm{~h}$ of thermotherapy 2 days a week for deep tumors. The gene therapy drugs were still administered periodically during treatment and radiotherapy can also join in the combination treatment.

Clinical trials. For those patients with advanced tumors and mutant $p 53$ gene detected, who have been clinically treated with a variety of therapies that are proved to be ineffective or are in need of alleviated symptoms, they can voluntarily participate in clinical trials for the expanded indications of rAd- $p 53$. It is believed that it can seek more therapeutic benefits through combination therapies including radiotherapy, chemotherapy, targeted drugs, immune agents, and others. More than 16 clinical trials of rAd-p53 have been performed during the last two decades in China. The pathologic types containing $\mathrm{HNSCC}^{30,49-51}$ non-small-cell lung cancer ${ }^{52,53}$ nasopharyngeal carcinoma, ${ }^{54-56}$ hepatic cell carcinoma $^{57-61}$, malignant gliomas ${ }^{62}$, and ovarian carcinoma, ${ }^{63}$ etc. These clinical trials demonstrated that rAd-p53 treatment groups exhibit better overall survival rates than control groups.

\section{EFFICACY EVALUATION AND ADVERSE EFFECTS}

\section{Efficacy evaluation}

The main objective of gene therapy completely differs from that of conventional therapies. The latter aims to get rid of the tumor by various means, such as surgery, chemotherapy, and radiotherapy. The main aim of chemoradiotherapy is to induce apoptosis by breaking DNA single and double strands, and destroying bases, and on the other hand, surgery is conducted to entirely eradicate tumor itself. ${ }^{64,65}$ Based on these therapeutic principles of conventional therapies, an international group collaborated to publish the Response Evaluation Criteria in Solid Tumors (RECIST) to evaluate treatment efficacy, which described when a tumor responds, when it remains stable, and when it progresses during the course of treatment. Comparing with the original evaluations, RECIST 1.1 is an evidence-based one, which evaluates more target lesions, highlights the necessity of the assurance of therapeutic effects, and updates the evaluation methods of lymph nodes. As neither the RECIST nor RECIST 1.1 can evaluate the effect of immune therapy appropriately, iRECIST has been developed. ${ }^{66,67}$

The fundamental goal of gene therapy is to turn the correct defective genes, which means to repair the genetic mutation or deletion, to bring an end to the immortal cancer cells, and to return them to their normal programmed cell death state. Because of the theoretical differences from the conventional therapies and immunotherapy, the evaluation of gene therapy should be different. It has been revealed that neither the RECIST, RECIST 1.1, nor the iRECIST was suitable for the therapeutic assessment. Treated with gene therapy combined with chemotherapy and/or radiotherapy, many patients are satisfied with good therapeutic effects. Although cancer foci can still be observed on CT or MRI, fewer subjective symptoms are manifested and overall survival is remarkably improved, and it is reasonably believed that the 
patients can live with tumors for a longer time. ${ }^{30}$ These better results are closely related to the principle of gene therapy, which is to replace tumor suppressor genes with normal and functional genes, rather than kill the entire tumor cell. Therefore, in our clinical practice, we cannot observe a rapid reduction in the size of the primary or metastatic cancer foci at the early stage of the combination therapies, but patients' symptoms greatly improved, which is not same as what is observed in the conventional treatment strategies. Based on the above experience, we reckon that the RECIST is not appropriate as the evaluation criteria for gene therapy and thus new efficacy evaluation guidelines should be developed for future clinical trials of gene therapy.

With the p53 gene transfected into tumor cells, they are "civilized" and display weakened malignant biological capabilities. Hence, human rAd-p53 treatment for malignant tumors is expected to be the most promising therapeutic approach to achieve long-term "live with tumor."

\section{Adverse effects}

The clinical application of human rAd-p53 is rather safe, with few serious adverse effects. The most common treatment-related adverse reaction is transient flu-like symptoms, clinically manifested by muscle soreness and fever (body temperature around $38^{\circ} \mathrm{C}$ ) on the day of administration. The rise in temperature is self-limited and the symptoms start to subside the next day without treatment. In cases of persistent high fever, physical cooling (alcohol baths) or medication (Nimesulide $25-50 \mathrm{mg}$ ) can be applied to reduce fever.

A rare complication is platelet crisis, featured by a sharp drop in platelet count below $30 \times 10^{9} / \mathrm{L}$ after administration and severe spontaneous bleeding. It should be treated promptly by blood transfusion, immunosuppressants, and hemostatic drugs, otherwise the patient's life will be endangered. Platelet crisis may be associated with immune dysfunction of gene therapy vectors, which can be effectively avoided by strictly grasping contraindications and carefully assessing patients' biochemical indexes and ECOG physical status before treatment.

\section{CONCLUSIONS}

At present, rAd-p53, a novel gene therapy reagent, has been widely applied to treat HNSCC, and been proved safe and effective in clinical practice. Based on gene therapy-related clinical trials and specialist experience, we provided and summarized the guidance on the clinical application of rAd-p53 for better facilitate the management of solid malignancies.

Both indications and contraindications of rAd-p53 are explicitly stated in this guidance, yet the former is not quite absolute. There are two major routes of administration: local intra-tumoral injection and arterial infusion (via superficial temporal artery), whereas intravenous administration should not be adopted. Moreover, the recommended dosage per administration of local intra-tumoral injection should be no less than $1 \times 10^{11} \mathrm{vp} / \mathrm{cm}^{3} / \mathrm{ml}$. According to the half-life of $\mathrm{rAd}-\mathrm{p} 53$, the whole treatment scheme should contain at least 5 administration cycles (every $72 \mathrm{~h}$ ) in order to attain favorable outcomes. In addition, when the clinical application of $\mathrm{rAd}-p 53$ as the gene therapy drug is in combination with radiotherapy, chemotherapy, or thermotherapy, it is possible to kill tumor cells to the maximum extent while preserving normal tissues, thus enhancing the therapeutic efficacy. Nevertheless, the evaluation of gene therapy does not coincide with that of traditional therapeutic strategies. Therefore, the future direction may fall on establishing and developing a novel evaluation system for the efficacy and safety of gene therapy.

\section{ACKNOWLEDGEMENTS}

The study was supported by National Natural Science Foundation of China (Grant numbers 81972538 and 81972546 ).

\section{ADDITIONAL INFORMATION}

Competing interests: The authors declare no competing interests.

\section{REFERENCES}

1. Brody, H. Gene therapy. Nature 564, S5 (2018).

2. Zhang, W. W. et al. The first approved gene therapy product for cancer Ad-p53 (Gendicine): 12 years in the clinic. Hum. Gene Ther. 29, 160-179 (2018).

3. Huang, J. Current developments of targeting the p53 signaling pathway for cancer treatment. Pharmacol. Ther. 220, 107720 (2021).

4. Tang, R. \& Xu, Z. Gene therapy: a double-edged sword with great powers. Mol. Cell Biochem. 474, 73-81 (2020).

5. Hernandez Borrero, L. J. \& El-Deiry, W. S. Tumor suppressor p53: biology, signaling pathways, and therapeutic targeting. Biochim. Biophys. Acta Rev. Cancer 1876, 188556 (2021).

6. Bieging, K. T., Mello, S. S. \& Attardi, L. D. Unravelling mechanisms of p53-mediated tumour suppression. Nat. Rev. Cancer 14, 359-370 (2014).

7. Liu, J., Zhang, C. \& Feng, Z. Tumor suppressor p53 and its gain-of-function mutants in cancer. Acta Biochim Biophys. Sin. (Shanghai) 46, 170-179 (2014).

8. Pitolli, C. et al. p53-mediated tumor suppression: DNA-damage response and alternative mechanisms. Cancers (Basel) 11, 1983 (2019).

9. Schweikl, H. et al. 2-Hydroxyethyl methacrylate-induced apoptosis through the ATM- and p53-dependent intrinsic mitochondrial pathway. Biomaterials 35, 2890-904 (2014).

10. Jiang, L. et al. Ferroptosis as a p53-mediated activity during tumour suppression. Nature 520, 57-62 (2015).

11. Chang, C. J. et al. p53 regulates epithelial-mesenchymal transition and stem cell properties through modulating miRNAs. Nat. Cell Biol. 13, 317-323 (2011).

12. Kim, N. H. et al. A p53/miRNA-34 axis regulates Snail1-dependent cancer cell epithelial-mesenchymal transition. J. Cell Biol. 195, 417-433 (2011).

13. Sachdeva, M. et al. p53 represses c-Myc through induction of the tumor suppressor miR-145. Proc. Natl Acad. Sci. USA 106, 3207-12 (2009).

14. Mello, S. S. et al. A p53 super-tumor suppressor reveals a tumor suppressive p53Ptpn14-Yap axis in pancreatic cancer. Cancer Cell 32, 460-473.e6 (2017).

15. White, E. Autophagy and p53. Cold Spring Harb. Perspect. Med. 6, a026120 (2016).

16. Mello, S. S. \& Attardi, L. D. Deciphering p53 signaling in tumor suppression. Curr. Opin. Cell Biol. 51, 65-72 (2018).

17. Pfaff, M. J. et al. Tumor suppressor protein $\mathrm{p} 53$ negatively regulates ischemiainduced angiogenesis and arteriogenesis. J. Vasc. Surg. 68, 222S-233S.e1 (2018).

18. Rufini, A. et al. Senescence and aging: the critical roles of p53. Oncogene $\mathbf{3 2}$, 5129-5143 (2013).

19. Kalanxhi, E. \& Dahle, J. The role of serotonin and p53 status in the radiationinduced bystander effect. Int. J. Radiat. Biol. 88, 773-776 (2012).

20. Uehara, I. \& Tanaka, N. Role of p53 in the regulation of the inflammatory tumor microenvironment and tumor suppression. Cancers (Basel) 10, 219 (2018).

21. Muñoz-Fontela, C. et al. Emerging roles of $\mathrm{p} 53$ and other tumour-suppressor genes in immune regulation. Nat. Rev. Immunol. 16, 741-750 (2016).

22. Dunbar, C. E. et al. Gene therapy comes of age. Science 359, eaan4672 (2018).

23. Misra, S. K. \& Bhattacharya, S. Physical chemical and biomolecular methods for the optimization of cationic lipid-based lipoplexes in vitro for the gene therapy applications. Methods Mol. Biol. 1445, 3-17 (2016).

24. Bottermann, M. et al. TRIM21 mediates antibody inhibition of adenovirus-based gene delivery and vaccination. Proc. Natl Acad. Sci. USA 115, 10440-10445 (2018).

25. Sun, W. et al. Advances in the techniques and methodologies of cancer gene therapy. Disco. Med. 27, 45-55 (2019).

26. $\mathrm{Li}, \mathrm{Y}$. et al. Key points of basic theories and clinical practice in rAd-p53 (Gendicine ${ }^{\mathrm{TM}}$ ) gene therapy for solid malignant tumors. Expert Opin. Biol. Ther. 15, 437-454 (2015).

27. Zhang, S.-Y., Lu, Y.-Y. \& Peng, Z.-H. In Recent Advances in Cancer Research and Therapy (eds Liu, X.-Y., Pestka, S., \& Shi, Y.-F.) 215-243 (Elsevier, 2012).

28. Zhang, W. W. Adenoviral vectors: development and application. Expert Opin. Investig. Drugs 6, 1419-1457 (1997).

29. Hendrickx, R. et al. Innate immunity to adenovirus. Hum. Gene Ther. 25, 265-84 (2014).

30. Li, Y. et al. Selective intra-arterial infusion of rAd-p53 with chemotherapy for advanced oral cancer: a randomized clinical trial. BMC Med. 12, 16 (2014).

31. Su, X. et al. Effect and safety of recombinant adenovirus-p53 transfer combined with radiotherapy on long-term survival of locally advanced cervical cancer. Hum. Gene Ther. 27, 1008-1014 (2016).

32. Kunimura, N. et al. Combination of rAd-p53 in situ gene therapy and anti-PD-1 antibody immunotherapy induced anti-tumor activity in mouse syngeneic urogenital cancer models. Sci. Rep. 10, 17464 (2020).

33. Reid, T., Warren, R. \& Kirn, D. Intravascular adenoviral agents in cancer patients: lessons from clinical trials. Cancer Gene Ther. 9, 979-986 (2002). 
34. Nastiuk, K. L. \& Krolewski, J.J. Opportunities and challenges in combination gene cancer therapy. Adv. Drug Deliv. Rev. 98, 35-40 (2016).

35. Hafner, A. et al. The multiple mechanisms that regulate p53 activity and cell fate. Nat. Rev. Mol. Cell Biol. 20, 199-210 (2019).

36. Ou, H. L. \& Schumacher, B. DNA damage responses and p53 in the aging process. Blood 131, 488-495 (2018).

37. Chen, J. The cell-cycle arrest and apoptotic functions of p53 in tumor initiation and progression. Cold Spring Harb. Perspect. Med. 6, a026104 (2016).

38. Li, T. et al. Tumor suppression in the absence of p53-mediated cell-cycle arrest, apoptosis, and senescence. Cell 149, 1269-1283 (2012).

39. Fischer, M. Census and evaluation of p53 target genes. Oncogene 36, 3943-3956 (2017).

40. Duffy, M. J., Synnott, N. C. \& Crown, J. Mutant p53 as a target for cancer treatment. Eur. J. Cancer 83, 258-265 (2017).

41. Goldstein, M. \& Kastan, M. B. The DNA damage response: implications for tumor responses to radiation and chemotherapy. Annu. Rev. Med. 66, 129-143 (2015).

42. O'Connor, M. J. Targeting the DNA damage response in cancer. Mol. Cell 60 , 547-560 (2015).

43. Talhouk, A. et al. Confirmation of ProMisE: a simple, genomics-based clinical classifier for endometrial cancer. Cancer 123, 802-813 (2017).

44. Parra-Herran, C. et al. p53, mismatch repair protein, and POLE abnormalities in ovarian clear cell carcinoma: an outcome-based clinicopathologic analysis. Am. J. Surg. Pathol. 43, 1591-1599 (2019).

45. Kane, J. R. et al. Sui generis: gene therapy and delivery systems for the treatment of glioblastoma. Neuro Oncol. 17, ii24-ii36 (2015).

46. Xie, Q. et al. Synergistic anticancer effect of exogenous wild-type p53 gene combined with 5-FU in human colon cancer resistant to 5-FU in vivo. World J. Gastroenterol. 22, 7342-7352 (2016).

47. Atencio, I. A. et al. Enhanced apoptotic activity of a p53 variant in tumors resistant to wild-type p53 treatment. Mol. Ther. 4, 5-12 (2001).

48. Rodriguez-Ramirez, C. \& Nör, J. E. p53 and Cell Fate: Sensitizing Head and Neck Cancer Stem Cells to Chemotherapy. Crit. Rev. Oncog. 23, 173-187 (2018).

49. Zhang, S. W. et al. Treatment of head and neck squamous cell carcinoma by recombinant adenovirus-p53 combined with radiotherapy: a phase II clinical trial of 42 cases. Zhonghua Yi Xue Za Zhi 83, 2023-2028 (2003).

50. Zhang, S. W. et al. [Recombinant adenovirus-p53 gene therapy combined with radiotherapy for head and neck squamous-cell carcinoma]. Zhonghua Zhong Liu Za Zhi 27, 426-428 (2005).

51. Liu, S. et al. Randomized, controlled phase II study of post-surgery radiotherapy combined with recombinant adenoviral human p53 gene therapy in treatment of oral cancer. Cancer Gene Ther. 20, 375-378 (2013).

52. Guan, Y. S. et al. Adenovirus-mediated wild-type p53 gene transfer in combination with bronchial arterial infusion for treatment of advanced non-small-cell lung cancer, one year follow-up. J. Zhejiang Univ. Sci. B 10, 331-340 (2009).

53. Wang, J. G. et al. [Treatment of local advanced non-small cell lung cancer with recombinant human p53 adenovirus combined with radiochemotherapy]. J. GuiYang Med. Coll. 39, 225-228 (2014).

54. Pan, J. J. et al. Effect of recombinant adenovirus-p53 combined with radiotherapy on long-term prognosis of advanced nasopharyngeal carcinoma. J. Clin. Oncol. 27, 799-804 (2009).

55. Liu, R. R., Ji, C. Y. \& Chen, J. C. Clinical effect of recombinant human p53 adv injection (gendicine) in combination with radiotherapy in patients suffering from recurrent nasopharyngeal carcinoma. J. Otolarngol. Ophthal. Shandong Univ. 24, 13-16 (2010).

56. $\mathrm{Si}, \mathrm{Y}$. F. et al. Recombinant adenovirus P53 agent injection combined with radiotherapy and chemotherapy for intermediate and advanced stage nasopharyngeal carcinoma. ZhongGuo Zhong Liu Lin. Chuang 36, 1031-1039 (2009).

57. Guan, Y. S. et al. p53 gene therapy in combination with transcatheter arterial chemoembolization for HCC: one-year follow-up. World J. Gastroenterol. 17, 2143-2149 (2011)

58. Tian, G. et al. Multiple hepatic arterial injections of recombinant adenovirus p53 and 5-fluorouracil after transcatheter arterial chemoembolization for unresectable hepatocellular carcinoma: a pilot phase II trial. Anticancer Drugs 20, 389-395 (2009).

59. Yang, Z. X. et al. Clinical study of recombinant adenovirus-p53 combined with fractionated stereotactic radiotherapy for hepatocellular carcinoma. J. Cancer Res. Clin. Oncol. 136, 625-30 (2010).

60. Ou, S. Q. et al. Recombinant adenovirus-p53 gene therapy combined with transcatheter arterial chemoembolization for p53-positive and p53-negative hepatocellular carcinoma. Chin. J. Inter. Imaging Ther. 7, 354-357 (2010).

61. Chen, S. et al. Clinical therapeutic effect and biological monitoring of p53 gene in advanced hepatocellular carcinoma. Am. J. Clin. Oncol. 37, 24-29 (2014).

62. Zhu, J. X. et al. Treatment of recurrent malignant gliomas by surgery combined with recombinant adenovirus-p53. Chin. J. Cancer Prev. Treat. 17, 126-128 (2010).

63. Cui, H. M. et al. Outcome of patients with recurrent epithelial ovarian carcinoma following treatment with recombinant human adenovirus p53 combined with chemotherapy. Chin. J. Cancer Biother 21, 450-454 (2014).

64. Li, Y. et al. A review of neoadjuvant chemoradiotherapy for locally advanced rectal cancer. Int. J. Biol. Sci. 12, 1022-1031 (2016).

65. Heinrich, S. \& Lang, H. Neoadjuvant therapy of pancreatic cancer: definitions and benefits. Int. J. Mol. Sci. 18, 1622 (2017).

66. Schwartz, L. H. et al. RECIST 1.1-update and clarification: From the RECIST committee. Eur. J. Cancer 62, 132-137 (2016).

67. Schwartz, L. H. et al. RECIST 1.1 - standardisation and disease-specific adaptations: Perspectives from the RECIST Working Group. Eur. J. Cancer 62, 138-145 (2016).

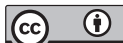

Open Access This article is licensed under a Creative Commons Attribution 4.0 International License, which permits use, sharing, adaptation, distribution and reproduction in any medium or format, as long as you give appropriate credit to the original author(s) and the source, provide a link to the Creative Commons license, and indicate if changes were made. The images or other third party material in this article are included in the article's Creative Commons license, unless indicated otherwise in a credit line to the material. If material is not included in the article's Creative Commons license and your intended use is not permitted by statutory regulation or exceeds the permitted use, you will need to obtain permission directly from the copyright holder. To view a copy of this license, visit http://creativecommons. org/licenses/by/4.0/.

(c) The Author(s) 2021 